Toute I'activité médicale tourne autour des patients, c'est pourquoi la FMH offre son soutien financier et idéologique au précieux travail accompli par la fondation Sécurité des Patients Suisse, notamment dans son projet actuel de Revues de morbidité et de mortalité (RMM). Le nouveau prix Innovation Qualité de l'Académie suisse pour la qualité en médecine (ASOM) de la FMH accorde également beaucoup d'importance à la sécurité des patients puisqu'il a créé, en collaboration avec Sécurité des Patients Suisse, une catégorie de prix spécifiquement dédiée à cette thématique. Si vous avez développé un projet dédié à la sécurité des patients et/ou à la qualité dans le domaine de la santé, alors saisissez cette occasion et inscrivez votre projet d'ici le 8 décembre 2017 au plus tard sur www.innovationqualite.ch.

\title{
Les revues de morbidité et de mortalité en Suisse
}

\author{
David Schwappach \\ Prof., responsable scientifique, Sécurité des Patients Suisse
}

Dans le secteur hospitalier, les revues de morbidité et de mortalité sont un outil largement utilisé pour favoriser l'apprentissage au niveau individuel et organisationnel. Ces colloques traitent des complications imprévues, déroulements inhabituels et décès inattendus. Sécurité des Patients Suisse a lancé, en collaboration avec la $\mathrm{FMH}$, un projet visant à renforcer les RMM en tant qu'outil pour promouvoir la sécurité des patients en Suisse.

Dans le monde anglo-saxon, les revues de morbidité et de mortalité font depuis longtemps partie de la formation postgraduée des médecins hospitaliers et sont également largement répandues dans les hôpitaux suisses. Initialement, ces colloques avaient pour vocation d'améliorer les pratiques professionnelles au travers d'une réflexion individuelle sur des cas dont le traitement était terminé. Depuis, cette perspective s'est modifiée, voire étendue.

\section{Perspective plus fortement orientée sur les systèmes}

De nombreux incidents et erreurs médicales découlent de l'organisation des soins, des processus, de la communication et des interactions. Fortes de ce constat, les RMM se sont plus spécifiquement orientées sur les systèmes. Dans un rapport du Kinderspital de Zurich, Frey et al. [1] montrent par ex. que les RMM interdisciplinaires et interprofessionnelles permettent d'identifier les erreurs dont une grande partie ont lieu au niveau des interfaces et de la collaboration au sein des équipes. Dans 37 des 48 RMM analysées (77\%), il a été possible d'identifier des erreurs ou un manque de sécurité dans les processus.

Des analyses internationales ont montré que les participants aux RMM apprécient cet outil qu'ils estiment primordial pour garantir la sécurité des patients. Dans les différentes enquêtes réalisées, les participants citent l'amélioration de la collaboration comme principal motif de participation à ces colloques [2]. L'adaptation des structures et des contenus vers un format plus orienté sur les systèmes a également été accueillie positivement [3].

Une implantation efficace des RMM est cependant exigeante. D'une part, la forme et la méthodologie doivent être adaptées, cela inclut notamment une réflexion fondamentale sur les systèmes. Les aspects formels, structurels, ainsi que le contenu et la méthodologie des RMM sont clairement liés à l'efficacité et à la réussite des mesures d'amélioration mises en place [4]. D’autre part, les conclusions, solutions et mesures élaborées dans le cadre des RMM doivent pouvoir trouver leur place dans l'institution, y être appliquées et porter leurs fruits.

La mise en place réussie des RMM exige des compétences sociales et méthodologiques de la part de la direction, de même qu'une culture d'entreprise qui permette de discuter ouvertement, en toute confiance et transparence, des événements indésirables ou des erreurs. A l'inverse, des revues de mortalité et de morbidité mal structurées et mal présentées peuvent avoir un effet délétère et mettre à mal une culture de la sécurité qui fonctionne bien. C'est notamment 
le cas si les personnes impliquées sont «montrées du doigt».

\section{Etat des lieux des RMM en Suisse}

Les RMM n'avaient jusqu'à présent pas encore été analysées en Suisse de manière systématique et peu d'outils pratiques et adaptés existent pour soutenir leur mise en place. C'est la raison pour laquelle Sécurité des Patients Suisse et la FMH ont lancé un projet visant à renforcer les RMM en tant qu'instrument pour promouvoir la sécurité des patients. Quels types de RMM existent en Suisse? Quelles expériences ont été faites et quelles améliorations sont nécessaires du point de vue des praticiens? Pour répondre à ces questions, un état des lieux des RMM a été dressé en Suisse grâce à une enquête en ligne lancée en 2017 auprès de tous les médecins-chefs exerçant dans des hôpitaux de soins aigus en Suisse (chirurgie, médecine interne, gynécologie et obstétrique, anesthésiologie et médecine intensive). Le résultat de cette enquête donne pour la première fois à la Suisse des données indiquant le point de vue des spécialistes sur l'avancement de la mise en œuvre des RMM, les attentes en matière de la poursuite de leur développement, les opportunités et les besoins à venir.

Les premières analyses montrent que les médecinschefs qui ont participé à l'enquête poursuivaient en premier lieu des objectifs pédagogiques et organisationnels visant à optimiser les processus et les déroulements, et que peu d'entre eux visaient à accroître les connaissances individuelles spécifiques de leurs collaborateurs. La perspective des RMM s'est également modifiée en Suisse et celles-ci sont de plus en plus axées sur les systèmes, alors que les nombreux aspects structurels et procéduraux recommandés au niveau international y trouvent encore peu d'écho, notamment la séparation entre la direction, l'animation et la présentation des RMM. L'établissement de critères clairs pour sélectionner les cas traités, la formation des animateurs ou la rédaction d'un procès-verbal anonyme pourtant recommandés, ne sont pas encore appliqués. Les données recensées montrent également de nettes différences entre les disciplines dans la manière dont les RMM sont réalisées et implantées.

\section{Nouveau souffle pour les RMM}

Bien que la plupart des participants estiment que les RMM sont un instrument efficace, le manque de temps,
Innovation Qualité: le prix destiné aux pionniers de la qualité

Sous le nom d'Innovation Qualité, I'Académie suisse pour la qualité en médecine (ASOM) de la FMH lance un nouveau prix visant à valoriser les projets qualité qui ont fait leurs preuves dans la pratique et à donner des impulsions novatrices au débat entre spécialistes. Le prix est décerné dans trois catégories, dont deux dotées de 15000 francs chacune, Repenser la prise en charge des patients et Sécurité des patients, et une dotée de 10000 francs, Organisations médicales. Soumettez dès à présent votre projet pour le prix Innovation Qualité! Le délai d'inscription est fixé au 8 décembre 2017 et la remise des prix aura lieu le 17 avril 2018. Vous trouverez de plus amples informations sous www.innovationqualite.ch

de disponibilité à s'engager et de compétences méthodologiques est fréquemment cité comme un obstacle à leur efficacité. La majorité des sondés voit pourtant un potentiel d'amélioration et de développement de «leur» RMM par le biais de listes de contrôle et de guides pour leur préparation. Ils souhaiteraient également disposer de modèles pour la rédaction de procès verbaux et la présentation et participer à des formations à l'animation. Sur la base des résultats de cette enquête, la fondation Sécurité des Patients Suisse s'attellera cet automne à élaborer de tels outils, car les conditions pour donner un second souffle à l'implémentation des RMM en tant qu'outil pour améliorer la sécurité des patients sont bonnes.

\section{Références}

1 Frey B, Doell C, Klauwer D, Cannizzaro V, Bernet V, Maguire C, et al. The Morbidity and Mortality Conference in Pediatric Intensive Care as a Means for Improving Patient Safety. Pediatric Critical Care Medicine 2016;17:67-72.

2 Lecoanet A, Vidal-Trecan G, Prate F, Quaranta JF, Sellier E, Guyomard A, et al. Assessment of the contribution of morbidity and mortality conferences to quality and safety improvement: a survey of participants' perceptions. BMC Health Serv Res 2016;16:1-7.

3 Murayama KM, Derossis AM, DaRosa DA, Sherman HB, Fryer JP. A critical evaluation of the morbidity and mortality conference. The American Journal of Surgery 2002;183:246-50.

4 François P, Prate F, Vidal-Trecan G, Quaranta JF, Labarere J, Sellier E. Characteristics of morbidity and mortality conferences associated with the implementation of patient safety improvement initiatives, an observational study. BMC Health Serv Res 2016;16:1-8. 